\title{
Primary Jejunal Adenocareinoma with Metastasis to Ovary in a Young Female
}

\author{
Ruchi Gaur', YK Saxena', S. Thomas ${ }^{2}$, S. Shukla ${ }^{3}$, Chitra R ${ }^{4}$ \\ From the Department of Radiotherapy ${ }^{1}$, Surgery ${ }^{2}$, Pathology ${ }^{3}$, Obstetrics and $^{2}$ \\ Gynaecology ${ }^{4}$, Lady Hardinge Medical College, New Delhi 110001 , India.
}

Abstract:

We report a rare case of primary jejunal adenocarcinoma with metastasis to ovary in a 23 year old female. She presented with four days' history of abdominal pain and vomiting. Barium meal follows through findings were suggestive of stricture for which resection was done. Pathological evaluation of resected specimen showed well differentiated adenocarcinoma grade-1, extending through the wall and adjoining mesentery. She was treated with adjuvant combination chemotherapy. Four months after completion of 6th chemotherapy cycle, she came with complaint of pain in abdomen. Physical examination and imaging studies revealed solid cystic lesion in right adenexal region. Excision of right tubo-ovarian mass, right salpingooopherectomy, and excision of previous jejuno-jejunal anastomosis with reanastomosis was done.

Histopathology and immunohistological staining of resected specimen revealed that tumor cells were positive for CK2O and CEA and negative for CK7 and CA125, favouring a diagnosis of metastatic carcinoma. The patient was again treated with oxaliplatin, 5-fluorouracil, and leucovorin based chemotherapy and recovered uneventfully. The case is reported in view of its clinical significance and rarity.

Key words: Adenocarcinoma, Jejunum, Ovary, Jejunal Neoplasms, Abdominal Pain.

\section{Introduction}

The small intestinal malignancies constitute $1-3 \%$ of all gastrointestinal malignancies, and $0.4 \%$ of all malignancies. The most common histological type is adenocarcinoma. It most frequently involves the duodenum, and with decreasing frequency, the jejunum and the ileum. Small intestine tumors are most commonly diagnosed after the $5^{\text {th }}$ decade of life with peak occurrence in the $6^{\text {th }}$ and $7^{\text {th }}$ decades. Most of the patients present with advanced disease. Metastases to omentum, liver and colon are common in these malignancies. Metastasis to ovary from the small bowel is extremely rare. We describe a case of 23 year old female who was diagnosed with primary jejunal adenocarcinoma, and subsequently developed secondary ovarian metastasis.

\section{Case Report}

A 23 year old female presented with four days' history of abdominal pain and vomiting. Her physical

Corresponding Author: Dr. Ruchi Gaur

Email: drruchisharma_77@yahoo.com

Received: October 8, 2013 | Accepted: November 9, 2013 | Published Online: November 25, 2013

This is an Open Access article distributed under the terms of the Creative Commons Attribution License (creativecommons.org/licenses/by/3.0)

Conflict of interest: None declared | Source of funding: Nil | DOl: http://dx.doi.org/10.17659/01.2013.0098 
examination revealed mild abdominal tenderness. Abdominal and pelvic ultrasound showed thickened bowel loops with stricture measuring $7 \mathrm{~mm}$, on the left side of abdomen. Upper $\mathrm{Gl}$ endoscopy showed no evidence of obstruction. Barium meal follows through findings were suggestive of stricture, which could be tuberculous or malignant in nature.

The patient was planned for surgery. Per-operative bowel examination revealed a stricture one foot distal to duodeno-jejunal junction with multiple enlarged mesenteric lymph nodes. Resection of jejunal stricture and excision of enlarged lymph nodes were done. Histological examination of resected specimen showed well differentiated adenocarcinoma grade-1, extending through the wall and adjoining mesentery. Isolated lymph nodes were negative for metastatic deposits. The patient was staged pT3NO and was planned for adjuvant chemotherapy with cisplatinum, 5 fluorouracil and leucovorin. During follow up, after 6 cycles of adjuvant chemotherapy CECT scan showed a mild anastomostic narrowing but no definitive mass was seen.

Four months after completion of the $6^{\text {th }}$ cycle of chemotherapy, the patient came with complaint of abdominal pain. On abdominal examination, a firm to hard lump measuring $10 \times 15 \times 15 \mathrm{~cm}$ was palpated on the right iliac and lumbar region. CECT scan of the abdomen and pelvis showed well defined multiseptate solid cystic lesion in right adenexal region extending upwards and displacing the loops of the small bowel [Fig.1]. Fat planes between adjacent bowel loops and mass were obliterated. Barium meal follow through findings showed mucosal folds at L3 vertebral level suggestive of jejuno-jejunal anastomosis. $\mathrm{X}$-rays chest was normal. CA 125 titre was $18 \mathrm{U} /$ $\mathrm{mL}$ (normal range, $<35 \mathrm{U} / \mathrm{mL}$ ). She was planned for exploratory laparotomy. Findings during the operation showed right tubo-ovarian mass $15 \times 15$ $\mathrm{cm}$ with lobulated surface. A thickening was present over previous jejuno-jejunal anastomosis. Multiple small lymph nodes were also present on adjacent mesentery. No omental or peritoneal deposits were seen. Liver, spleen, stomach and rest of the small and large bowel were normal on inspection and palpation. Based on these findings excision of right tubo-ovarian mass, right salpingo-oopherectomy, and excision of previous jejuno-jejunal anastomosis with re-anastomosis was carried out.

Histopathological examination of the resected ovarian specimen showed variable sized irregular glands lined by columnar epithelium showing nuclear hyperchromasia and loss of polarity with large areas of confluent necrosis [Fig.2]. Dirty necrosis within glandular lumina, focal segmental necrosis of glandular epithelium and garlanding of neoplastic glands around necrotic material were suggestive of metastatic adenocarcinoma. Immunohistochemistry revealed that tumor cells were positive for CK20 and CEA and negative for CK7 and CA1 25, favouring a diagnosis of metastatic ovarian adenocarcinoma [Fig. 3,4]. Sections from left ovary, jejunum, lymph nodes and omentum were free of tumor.

\section{Discussion}

The small intestine constitutes approximately $80 \%$ of the total length of the gastrointestinal tract and it makes up $90 \%$ of its absorptive surface yet the reported incidence of small bowel tumors is $1-3 \%$ of all gastrointestinal malignancies, and $0.4 \%$ of all malignancies $[1,2]$. The most common histological type is adenocarcinoma, followed by carcinoid, lymphoma, sarcoma and GISTs. Adenocarcinoma most frequently involves the duodenum, and with decreasing frequency, the jejunum and the ileum. It is most commonly diagnosed after $5^{\text {th }}$ decade of life with peak occurrence in the $6^{\text {th }}$ and $7^{\text {th }}$ decades [3-6]. Only eight cases of jejunum adenocarcinoma have been reported in patients under 20 years of age $[7,8]$. Most of the reported series have found equal sex distribution but others have reported 


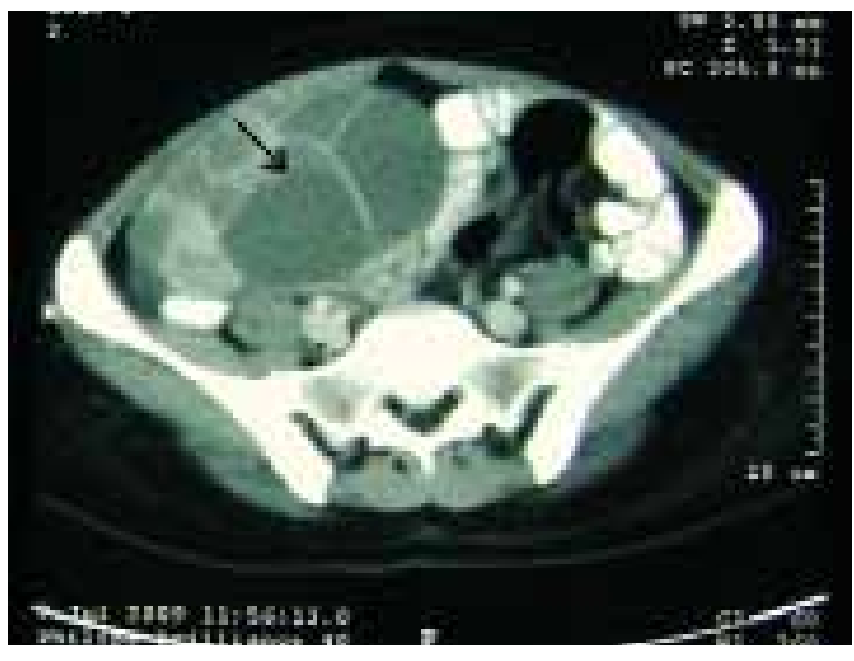

Fig.1: CECT image showing well defined multiseptate solid- cystic lesion in right adenexa.

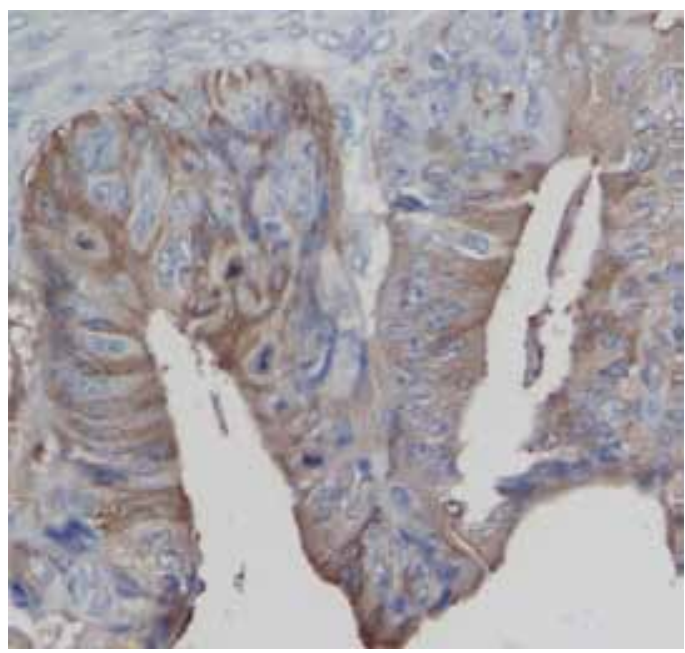

Fig.3: Photomicrograph of tumor cells showing immunoreactivity for carcinoembrionic antigen (IHC 400).

male preponderance [3-6]. Jejunal and ileal adenocarcinoma often present with vague and non specific symptoms that develop insidiously and determined by the tumor location, growth rate and size. These symptoms usually related to some degree of intestinal obstruction. The most common presenting complaints are abdominal pain, nausea, vomiting, weight loss, and change in bowel habits.

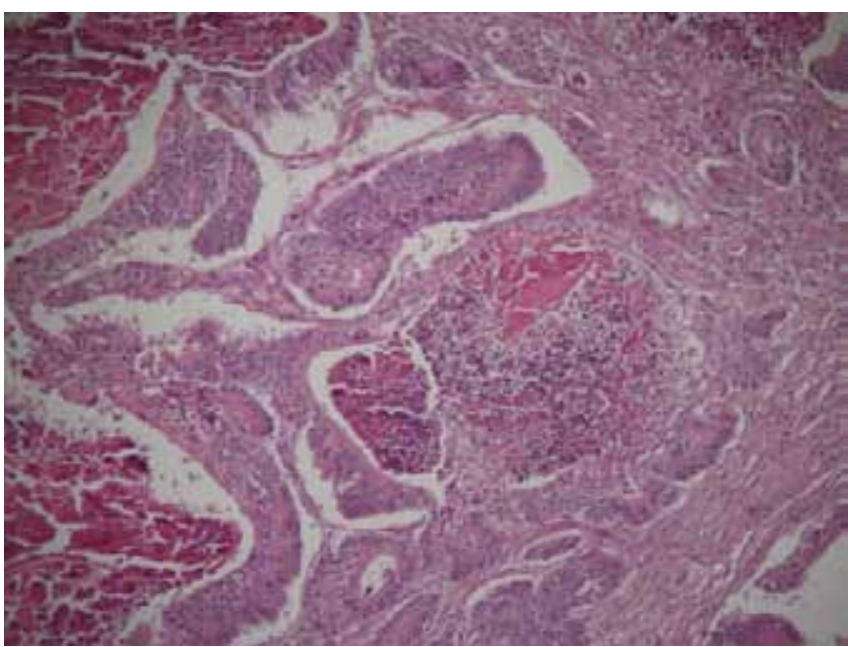

Fig.2: Photomicrograph showing variable sized neoplastic glands with large areas of necrosis (H\&E 100).

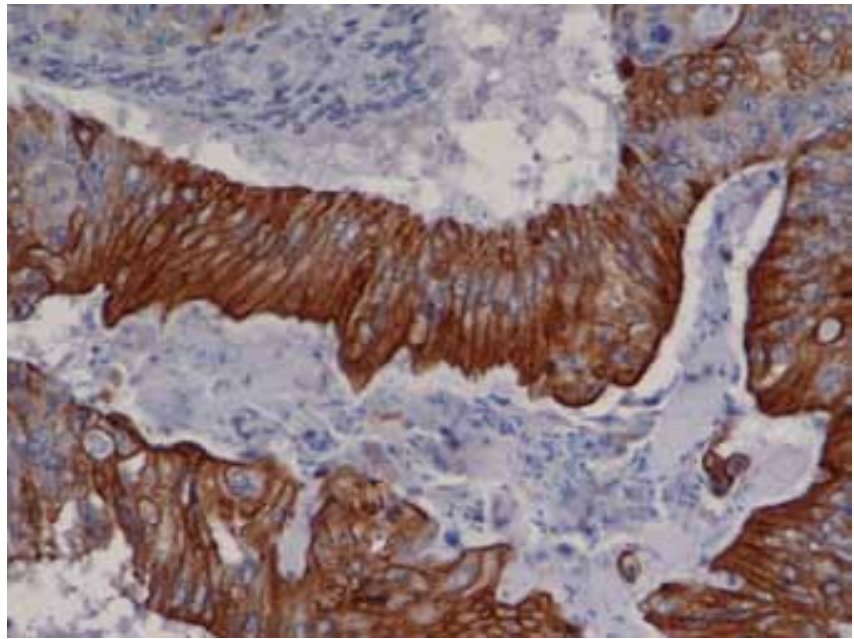

Fig.4: Photomicrograph showing neoplastic glands with strongly positive reaction for CK20 (IHC 400).

Non-specific signs and symptoms limit the early detection of primary jejunal carcinoma and most of the patients present with advanced disease. Treatment of choice for curative intent is surgery which includes en-bloc tumor resection including adherent structures. Advanced disease usually managed by by-pass surgery. There is no 
established protocol for adjuvant chemotherapy or radiotherapy for jejunal and ileal adenocarcinoma. Chemotherapy regimes designed for colon cancer have not been proven beneficial for small bowel neoplasm. Metastases are very frequently seen in these malignancies.

Metastatic ovarian neoplasia constitutes $3-8 \%$ of all ovarian tumors and $10-30 \%$ of malignant ovarian tumors [9]. The colon, stomach, and the breast are the most common extra genital sites, metastasizing to ovaries. Metastatic carcinoma to ovary from the small bowel is extremely rare. In all reported cases of adenocarcinoma of jejunum with ovarian metastasis patient had ovarian involvement at the time of presentation in contrast to this case. Three major routes of metastasis to ovary from the gastrointestinal tract are suggested in the literature i.e. retrograde lymphatic spread; via peritoneal fluid and haematogenous spread [10].

Review of literature showed that garland and cribriform growth pattern, intraluminal "dirty" necrosis, segmental destruction of glands and absence of metaplasia are characteristic microscopic features of ovarian metastasis from large intestine [11]. In our case histopathologic examination of resected specimen also showed dirty necrosis within glandular lumina, focal segmental necrosis of glandular epithelium and garlanding of neoplastic glands around necrotic material.

Immunohistochemistry is perhaps the only reliable method, which can guide to differentiate gastrointestinal tract and ovarian primaries. Various studies showed that colonic carcinoma is typically positive for CEA and CK2O and negative for CK7 and CA125. Study by Sack et al showed that all metastatic ovarian carcinoma were positive for CK7, and combined use of CK2O and CK7 antibodies is highly sensitive and specific for metastatic colonic carcinoma [12]. Lagendijk et al demonstrated that CK7 and CEA is best combination marker which differentiates between secondary's from ovary and colon [13]. In our case tumor cells showed strong positivity against CK20 and CEA and no reaction for CK7 and CA 125.

\section{Conclusion}

Primary jejunal malignancies are rare at young age and only six similar cases could be found on exhaustive literature search. In the past there were difficulties in making accurate diagnosis. In our report the role of immunohistochemical markers has been emphasized in reaching accurate diagnosis which helps in planning further management.

\section{References}

1. Jemal A, Siegel R, Ward E, Murray $T, X u$ J, Thun MJ. Cancer statistics, 2007. CA Cancer J Clin. 2007;57:43-66.

2. North JH, Pack MS. Malignant tumours of small intestine: a review of 144 cases. Am Surg. 2000;66:46-51.

3. MorganDF, Busuttil RW.Primaryadenocarcinoma of the small intestine. Am J Surg. 1977;134:331 333.

4. Miles RM, Crawford D, Duras S. The small bowel tumour problem: an assessment based on a 20 year experience with 116 cases. Ann Surg. 1979; 189:732-738.

5. Adler SN, Lyon DT, Sullivan PD. Adenocarcinoma of the small bowel: clinical features, similarity to regional enteritis, and analysis of 338 documented cases. Am J Gastroenterol. 1982;77:326-330.

6. Zar N, Holmberg L, Wilander E, Rastad J. Survival in small intestinal adenocarcinoma. Eur J Cancer. 1996;32A(12):21 14-2119.

7. Dunsmore KP, Lovell MA. Small bowel adenocarcinoma metastatic to the ovaries in a 12-year-old girl. J Pediatr Hematol Oncol. 1998;20:498-501.

8. Huang Chen-Sheng, Luo Chin-Cheng, Lin Jer 
Nan, Huang Shin-Feng. Adenocarcinoma of the jejunum: a pediatric case report. Chang Gung Med J. 2003; 26:204-207.

9. Nobuhiro Tsuruchi, Hitoshi Kubota, naoki Tsukamoto, Akihiko Kurano. Primary jejunal adenocarcinoma masquerading as a primary ovarian malignancy. Gynaecologic Oncology. 1995;58:129-132.

10. Chang TC, Changchien CC, Tseng CW, Lai CH, Tseng CJ, Lin SE, et al. Retrograde lymphatic spread: a likely route for metastatic ovarian cancers of gastrointestinal origin. Gynecol Oncol. 1997;66:372-377.

11. Lash RH,Hart,WR. Intestinal adenocarcinomas metastatic to the ovaries: A clinicopathologic evaluation of 22 cases. Am. J Surg Pathol. 1987;1 1:114-121.

12. Sack MJ, Roberts SA. Cytokeratin 20 and 7 in the differential diagnosis of metastatic carcinoma in cytologic specimens. Diagn Cytopathol. 1997; 16:132-136.

13. Lagendijk JH, Mullink $H$, Van Diest PJ, Meijer GA, Meijer CJ. Tracing the origin of adenocarcinomas with unknown primary using immunohistochemistry: differential diagnosis between colonic and ovarian carcinomas as primary sites. Hum Pathol. 1998;29:491-497. 